\title{
Valence, Not Utility, Underlies Reward-Driven Prioritization in Human Vision
}

\author{
Ludwig Barbaro, ${ }^{-}$Marius V. Peelen, and $\$ Clayton Hickey \\ Center for Mind/Brain Sciences, University of Trento, 38068, Rovereto, Italy
}

\begin{abstract}
Objects associated with reward draw attention and evoke enhanced activity in visual cortex. What is the underlying mechanism? One possibility is that reward's impact on vision is mediated by unique circuitry that modulates sensory processing, selectively increasing the salience of reward-associated stimuli. Alternatively, effects of reward may be part of a more general mechanism that prioritizes the processing of any beneficial object, importantly including stimuli that are associated with the evasion of loss. Here, we test these competing hypotheses by having male and female humans detect naturalistic objects associated with monetary reward, the evasion of equivalent loss, or neither of these. If vision is economically normative, processing of objects associated with reward and evasion of loss should be prioritized relative to neutral stimuli. Results from fMRI and behavioral experiments show that this is not the case: whereas objects associated with reward were better detected and represented in ventral visual cortex, detection and representation of stimuli associated with the evasion of loss were degraded. Representations in parietal cortex reveal a notable exception to this pattern, showing enhanced encoding of both reward- and loss-associated stimuli. Experience-driven visual prioritization can thus be economically irrational, driven by valence rather than objective utility.
\end{abstract}

Key words: attention; fMRI; incentive salience; MVPA; reward; vision

\section{Significance Statement}

Normative economic models propose that gain should have the same value as evasion of equivalent loss. Is human vision rational in this way? Objects associated with reward draw attention and are well represented in visual cortex. This is thought to have evolutionary origins, highlighting objects likely to provide benefit in the future. But benefit can be conferred not only through gain, but also through evasion of loss. Here we demonstrate that the visual system prioritizes real-world objects presented in images of natural scenes only when these objects have been associated with reward, not when they have provided the opportunity to evade financial loss. Visual selection is thus non-normative and economically irrational, driven by valence rather than objective utility.

\section{Introduction}

Visual stimuli associated with reward become salient and attentiondrawing. As a result, they are easy to find as targets of visual search but hard to ignore when they act as task-irrelevant distractors (for review, see Anderson, 2016; Le Pelley et al., 2016). This selective bias sustains when it is counterproductive (Hickey et al., 2010) and appears to rely at least in part on plasticity in visual cortex (van Koningsbruggen et al., 2016), suggesting that it is a product of implicit attentional and perceptual learning rather than ex-

\footnotetext{
Received April 25, 2017; revised Sept. 1, 2017; accepted Sept. 18, 2017.

Author contributions: L.B., M.V.P., and C.H. designed research; L.B. performed research; L.B., M.V.P., and C.H. analyzed data; L.B., M.V.P., and C.H. wrote the paper.

This work was supported by the Autonomous Province of Trento, Italy (Grandi Progetti 2012 project, Characterizing and improving brain mechanisms of attention-ATTEND).

The authors declare no competing financial interests.

Correspondence should be addressed to Dr. Clayton Hickey, Center for Mind/Brain Sciences, University of Trento, Corso Bettini 31,38068, Rovereto, Italy. E-mail: clayton.hickey@unitn.it.

DOI:10.1523/JNEUROSCI.1128-17.2017

Copyright $\odot 2017$ the authors $\quad 0270-6474 / 17 / 3710438-13 \$ 15.00 / 0$
}

plicit strategy. It may have evolutionary origins (facilitating the search for food), and could underlie maladaptive selective behavior observed in eating disorders and addiction (Robinson and Berridge, 1993; Berridge, 1996).

This effect of reward on selection has been interpreted in two ways. On one hand is the idea that reward may activate unique and specific brain mechanisms that prioritize reward-predictive stimuli in visual processing (Hickey et al., 2010; Roelfsema et al., 2010). This is in line with ideas from the animal literature about approach behavior and the function of reward-related dopamine (Berridge and Robinson, 1998), and is reflected in a recent computational model that proposes long-range neurochemical interactions between the reward system and visual cortex (Roelfsema and van Ooyen, 2005). But, on the other hand is the equally compelling idea that attentional bias to reward-associated stimuli might constitute one instantiation of a broader motivational mechanism in vision (Gottlieb, 2012; Le Pelley et al., 2016). Using a term from economics, this would act to maximize objective utility, the absolute quality or usefulness of outcome, by priori- 


\section{Utility Model}
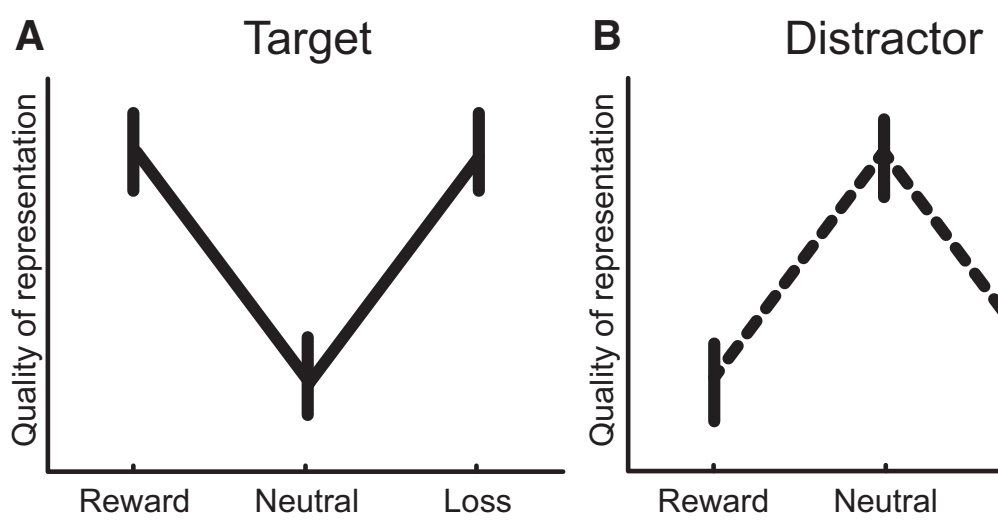

Valence Model
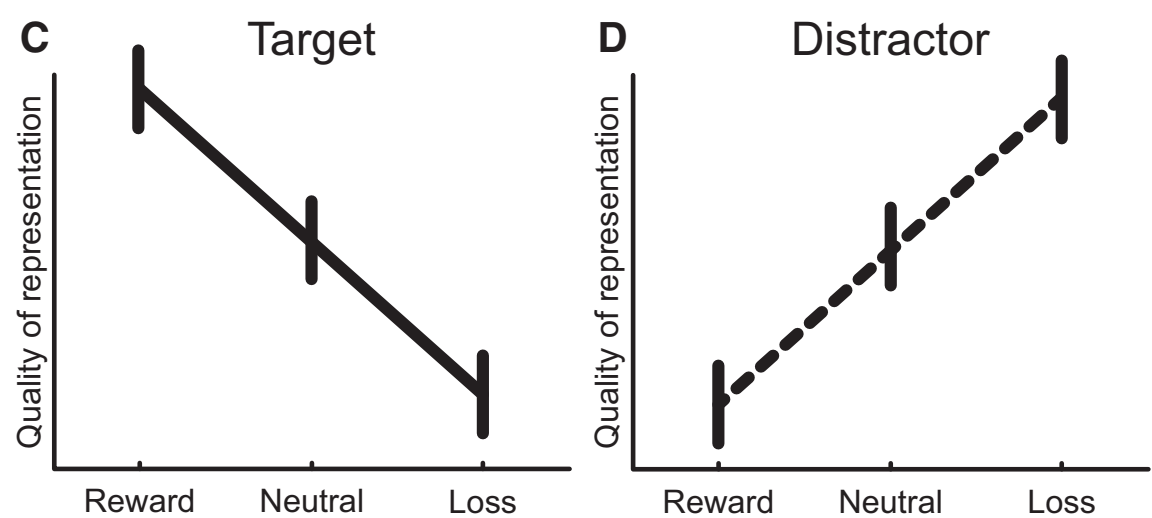

Figure 1. Predictions from the utility and valence models. $A$, By the utility model, reward-and loss-associated targets should draw selective resources, and thus be better represented than neutral targets. $\boldsymbol{B}$, During search for neutral targets, reward- and loss-associated distractors should require the same degree of attentional suppression, and thus be poorly represented relative to neutral distractors. $C$, By the valence model, reward-associated targets should draw selective resources, whereas loss-associated targets may be actively suppressed and poorly represented in the visual system. $\boldsymbol{D}$, Reward-associated distractors should require attentional suppression, but loss-associated distractors should not.

tizing any informative object that can be acted on to create a relative benefit, including stimuli that predict negative outcome when this information can be used to evade greater loss or harm.

Here we test these accounts of reward's influence on visual selection. We designed a visual search task in which participants could accumulate points with cash value by correctly detecting examples of target categories in pictures of natural scenes. A given category could be associated with rewarding outcome, neutral outcome, or loss. Correct detection of an example of the rewardassociated category thus resulted in receipt of 150 points, but failure to detect this target garnered only 50 points. In contrast, correct detection of an example from the loss-associated category resulted in the forfeiture of 50 points, but failure to detect this target resulted in the greater loss of 150 points. Finally, detection of a neutral target garnered only 1 point, and failure to detect this target resulted in the loss of only 1 point. Accurate detection of both reward- and loss-associated targets in our design therefore had a consistent benefit of 100 points relative to incorrect performance, making them more valuable than neutral targets, and participants were made explicitly aware of this fact.

If selective bias for reward-associated stimuli reflects a rational, utility-maximizing mechanism, reward- and lossassociated targets should be prioritized equally in our task. This motivates the set of predictions illustrated in Figure $1 A, B$, which we refer to as the utility model. Targets should draw attention and be well represented in visual cortex (Hickey et al., 2010; Anderson et al., 2011), but reward- and loss-associated targets should draw attention with greater strength than neutral targets (see Fig. 1A). When these objects act as task-irrelevant distractors, their salience will cause them to interfere with search for other targets and they will need to be attentionally suppressed (Hickey and Peelen, 2015, 2017; Sawaki et al., 2015) (see Fig. 1B). But if selective bias for reward-associated stimuli is not economically rational, it presumably reflects an impact of the absolute valence of outcome. By this valence model, targets that predict reward should be visually salient (see Fig. 1C), and reward-associated distractors should require strong attentional suppression (see Fig. 1D). Targets predicting suboptimal outcome, however, may not only fail to draw attention but be actively suppressed and poorly represented in the visual system (Hickey et al., 2010; Hickey and van Zoest, 2012; Hickey and Peelen, 2017) (see Fig. 1C). If this is so, loss-associated distractors may not interfere with search, thus requiring little inhibition (see Fig. 1D).

\section{Materials and Methods}

We conducted two experiments to test the predictions illustrated in Figure 1. Experiment 1 relied on the use of multivariate pattern analysis (MVPA) of human fMRI data to index the quality of visual representations in the brain. While in the scanner, 23 participants searched for examples of real-world object categories in images of city and landscapes (see Fig. 2A). The target category (cars, trees, buildings, or people) was indicated at the beginning of each block of trials, and for each participant one object category was associated with reward when it acted as target, one to loss, and the others to neutral outcome (see Fig. 2B). Importantly, when search was for one of the neutral categories, examples of the rewardassociated, loss-associated, and other neutral category appeared in the scenes as irrelevant distractors.

To maximize our power to detect potentially subtle effects on distractor representations, Experiment 1 adopted a design where examples of all distractor categories were present in every scene. We therefore conducted a second behavioral experiment to determine how the association of reward or loss to distractors impacted search behavior. Here, 101 participants completed a variant of the task in which outcome was manipulated independently of target identity. Each block thus began with a cue indicating both target category and pay scheme and scenes only occasionally contained outcome-associated distractors. By looking at performance within each block, we were able to examine how outcome association impacted target detection, whereas by looking across blocks we were able to see how examples from object categories that had recently been associated with reward or loss came to interfere with detection of neutral targets.

Participants. Twenty-four volunteers ( 9 female; mean \pm SD age, $25 \pm$ 4 years) gave informed consent before completing Experiment 1 . One participant reported the target as present in $>90 \%$ of trials and was excluded from analysis. A total of 105 volunteers gave informed consent before completing Experiment 2 ( 79 female, $23 \pm 4$ years). Three partic- 
ipants were excluded because of low accuracy ( $>3$ SD from group mean) and one because of inconsistent responses on a screening questionnaire. For both experiments, sample size was guided by observed effect sizes in our prior work using similar methodology. All methods were approved by the University of Trento ethical committee and adhered to the Declaration of Helsinki.

Experiment 1: object selective cortex (OSC) localizer. The OSC localizer involved 2 scanner runs of $317 \mathrm{~s}$ duration, each comprised of 16 blocks of 20 trials and 3 fixation blocks. Each run started with a 15 s fixation period followed by a $2 \mathrm{~s}$ reminder of task instructions and ended with a $15 \mathrm{~s}$ fixation period. Each trial began with fixation $(400 \mathrm{~ms})$ followed by a central image of an isolated everyday object (e.g., telephone, alarm clock, blender; $350 \mathrm{~ms}, n=20$ ) or a pixel-scattered version of one of these images. Participants monitored for image repetition, which occurred once per block. Every fifth block was a fixation block, where for $15 \mathrm{~s}$ only the fixation cross was presented.

Experiment 1: category pattern localizer. The category pattern localizer involved 2 scanner runs of $392 \mathrm{~s}$ duration, each comprised of 20 blocks of 20 trials and 4 fixation blocks. Each run started with a $15 \mathrm{~s}$ fixation period followed by a $2 \mathrm{~s}$ reminder of task instructions and ended with a $15 \mathrm{~s}$ fixation period. In each block, images of isolated examples of one of the four relevant stimuli categories (cars, trees, buildings, or people) were presented ( $n=40$ per category). Each trial began with fixation ( $400 \mathrm{~ms}$ ) followed by image presentation $(350 \mathrm{~ms})$. As in the OSC localizer, participants monitored for image repetition, which occurred once per block, and every fifth block was a fixation block where for $15 \mathrm{~s}$ only the fixation cross was presented. Localizer images of people were headless because faces in the primary experimental task were commonly too small to visually resolve. We did not want the localizer pattern to reflect the encoding of faces if this was not possible in the experiment itself.

As an internal check, we conducted a split-half correlation analysis of category patterns in OSC. This correlated the pattern for each category as derived from half the data with the pattern for each category as derived from the other half of the data. Patterns for the same categories were similar, and thus positively correlated on average $(0.73)$, whereas the patterns across categories were dissimilar and thus negatively correlated $(-0.23)$.

Experiment 1: design. The primary experiment involved 4 scanner runs of 590 s duration, each comprised of 8 blocks of 24 trials. Each run started and ended with a $15 \mathrm{~s}$ fixation period. At the beginning of each block, central text indicated the target category for the coming block for $10 \mathrm{~s}$ (cars, trees, buildings, people) alongside the number of points that had been accumulated to that point in the experiment. The trial sequence is illustrated in Figure 2A. In half of the trials, the scene contained examples of all relevant object categories. In remaining trials, the target was absent, and the order of target-present and target-absent trials was randomized within each block. Participants reported the presence or absence of the target with the right index and middle fingers, respectively. Late responses $(>750 \mathrm{~ms})$ and missing responses were incorrect. When the target was present and response was accurate, feedback was determined by the target category (see Fig. 2B). The association of specific categories to reward, loss, and neutral outcome was counterbalanced across participants, and participants were explicitly informed at the beginning of the experiment about the relationship between each target category and its associated outcome. All participants completed a short training procedure before entering the scanner and were able to report this relationship before beginning the experiment itself. Participants were paid based on the number of points they accumulated during the experiment $(€ 0.0018 /$ point), and each received between $€ 25$ and $€ 40$.

Experiment 1: fMRI data acquisition, preprocessing, and analysis. Whole-brain Imaging was conducted using a Bruker BioSpin MedSpec 4T scanner with an eight-channel head coil (functional data: $2^{\star}$-weighted echo-planar images, 31 slices with $0.45 \mathrm{~mm}$ gap, $3 \times 3 \times 3 \mathrm{~mm}$ voxel size, $\mathrm{TR}=2.2 \mathrm{~s}, \mathrm{TE}=33 \mathrm{~ms}$, flip angle $=76^{\circ}$; structural data: $\mathrm{T} 1$-weighted MPRAGE, 256 slices, $1 \times 1 \times 1 \mathrm{~mm}$ voxel size). Functional data were motion corrected, slice time corrected, smoothed using a $6 \mathrm{~mm}$ FWHM Gaussian kernel, high-pass filtered at $0.008 \mathrm{~Hz}$, coregistered to the structural image, and spatially normalized to the MNI template. Preprocessing and subsequent data analysis were conducted with SPM12
(University College London) and the CoSMo-MVPA toolbox (Oosterhof et al., 2016).

A separate GLM was created for each of the OSC localizer, category localizer, and visual search task. In each GLM, all regressors of interest were convolved using the canonical hemodynamic response function implemented in SPM12, and all models contained six regressors of no interest obtained from the realignment procedure to account for head motion.

In the OSC localizer, the BOLD signal for each voxel was modeled using two regressors of interest: one for intact and one for scrambled objects. OSC was functionally defined in MNI space for each participant by contrasting responses evoked by the intact and scrambled images and identifying reliably activated voxels in occipital or temporal cortex $(p<$ 0.001 , uncorrected). Mean OSC size was $1491 \pm 845$ voxels. In the category localizer, four regressors of interest were used, reflecting the presentation of people, cars, buildings, or trees. In the visual search task, four regressors of interest were used, reflecting the block target. All regressors of interest spanned whole experimental blocks.

Experiment 1: MVPA. Category patterns were derived from the category localizer data by identifying the set of $t$ values elicited for each voxel in OSC by each of the four object categories. Similar patterns were isolated for each condition of interest in the visual search experiment. All voxelwise $t$ values were normalized within each experiment by subtracting the mean $t$ value observed across experimental conditions from each conditional value (Haxby et al., 2001). This removes voxel variance unrelated to experimental manipulations while retaining conditional variance. OSC patterns observed in the visual search experiment were subsequently correlated with OSC patterns observed in the category localizer to generate a $4 \times 4$ correlation matrix. These values were Fishertransformed and organized in terms of category outcome association and whether the category acted as target or distractor. As an internal check, we determined that OSC patterns elicited by scenes correlated more strongly with the benchmark pattern corresponding to the target $($ mean $=0.125)$ than they did with the other benchmark patterns $(-0.041 ; p<0.001)$.

The correlation values computed in this way are on an interval rather than a ratio scale, with a zero point that is not informative. This is the case because of the normalization procedure (Haxby et al., 2001; Misaki et al., 2010). When all pairwise correlations are calculated for the conditions of two individually normalized datasets, the resulting set of normalized correlations are centered on zero. Negative values are thus negative only in relationship to the mean of all possible cross-correlations between the datasets. Raw, non-normalized correlation values in Experiment 1 were consistently positive because scene and localizer stimuli evoked a similar visual response in many OSC voxels. The normalization procedure isolated conditional differences in this signal.

Each category type acted as distractor in three block types. For example, if a category was associated with neutral outcome, it could act as distractor when the target category was also neutral, when the target category was associated with reward, and when the target category was associated with evasion of loss. However, to isolate changes in distractor representation that were produced solely by the association of specific outcome to the distractor category, we focused analysis of distractor categories on results observed when the target was associated with neutral outcome. The general pattern of distractor information emerges in much the same way if analysis includes conditions where the target could be associated with reward or evasion of loss.

Experiment 1: regression analysis. To statistically assess the ability of the utility and valence models to account for our OSC and searchlight results, we conducted multiple linear regression analyses for each participant dataset (and, in the case of searchlight analysis, for each searchlight sphere). The first regressor described the difference in task relevance between targets and distractors, positively weighting targets and negatively weighting distractors (i.e., $1,1,1,-1,-1,-1$ ). The second described the valence model, positively weighting reward-associated targets and loss-associated distractors and negatively weighting loss-associated targets and reward-associated distractors (i.e., $1,0,-1,-1,0,1)$. The final regressor described the utility model, positively weighting both reward- and loss-associated targets and negatively weighting both rewardand loss-associated distractors (i.e., $1,-2,1,-1,2,-1$ ). Each regressor 
vector is orthogonal to the others and has a mean value of zero, and distractor weights are an inverted version of target weights. Regressor vectors were $z$-scored before linear regression was conducted. This analysis garnered a coefficient for each participant that was positive in value when the regressor predicted dataset variance. The sets of coefficient values observed across participants were tested against a null hypothesis of zero.

Experiment 1: correlation between univariate activity and information. We approached the experiment with the idea that reactivity in the dopaminergic midbrain might predict the quality of object representation in OSC (Hickey and Peelen, 2015, 2017). To test this hypothesis, we began by conducting a separate event-related GLM analysis with a predictor for the presence of reward-associated, loss-associated, and neutral targets. We subsequently used a probabilistic anatomical MRI atlas (Maldjian et al., 2003) to define two midbrain regions of interest (ROIs), one describing the bilateral red nuclei and the other the bilateral substantia nigra (SN) (see Fig. 4A). We extracted activity in each of these ROIs as observed when a reward-associated, loss-associated, and neutral target was present in the scene. Following the logic of our regression analysis of OSC category information, we conducted a regression analysis of this univariate activity with predictors for the utility (i.e., $1,-2,1$ ) and valence models (i.e., $1,0,-1$ ).

This analysis, and the preceding GLM, was limited to activation elicited by the presence of targets because all distractors were consistently present in each trial in the design of Experiment 1. To maintain equivalence between univariate and multivariate results, we calculated new regression coefficients for OSC category information with utility and valence predictors modeling the representation of only targets (and thus ignoring variance in the representation of distractors). The target-only univariate midbrain coefficients were then correlated with these targetonly category information coefficients (see Fig. $4 B$ ).

We conducted a whole-brain analysis based on the same logic as this ROI-based analysis (Hickey and Peelen, 2015). For each voxel, coefficients for the valence model were calculated based on univariate activity observed in that voxel in response to scenes containing reward-associated, loss-associated, and neutral targets. The resulting values were correlated across participants with target-only category information coefficients. These correlation values were Fisher transformed and assigned to the voxel location in a new brain volume.

Experiment 1: whole-brain searchlight analysis. Searchlight analysis began with the identification of brain regions where scene-evoked patterns contained more information about targets than distractors. For each voxel in the brain, we defined a surrounding sphere with $21 \mathrm{~mm}$ diameter (7 voxels). We subsequently computed voxelwise correlations between scene-evoked activity in each of these spheres and spatially equivalent mean benchmark patterns evoked in our category pattern localizer. These correlation values were Fisher transformed and assigned to the center voxel of each sphere. Target category information (i.e., the correlation between the scene-evoked pattern and the benchmark pattern for the object category currently acting as target in the scene) was subsequently contrasted with nontarget category information (i.e., the correlation between the scene-evoked pattern and the benchmark pattern for the object categories currently acting as distractors in the scene). For each voxel, this difference was tested for statistical reliability across participants.

Voxels showing selectivity at $p<0.001$ were selected for further analysis when they were part of a cluster with minimum size of 50 voxels. This identified three clusters. Figure $5 \mathrm{~A}$ illustrates OSC, as identified in our OSC localizer experiment. The first and second clusters identified in this searchlight analysis are illustrated in Figure $5 B$, and are approximately equivalent in size and location to bilateral OSC. The third cluster was located in posterior parietal cortex and is illustrated in Figure $6 \mathrm{~A}$.

Further analysis was constrained to the parietal cluster, where we examined variance in category information in each sphere as a function of outcome association. To test the degree to which category information for each sphere followed the utility or valence model, we isolated category information for reward-associated, loss-associated, and neutral category examples when these were the target of search, and for reward-associated, loss-associated, and neutral category examples when these were distrac- tors and the target of search was an example of one of the two outcomeneutral object categories. We conducted multiple linear regression for category information at each voxel using the same regressor weights described above. All regression coefficient values corresponding to voxels falling within the parietal ROI were mean averaged to generate a single value describing the explanatory power of the task relevance, utility, and valence models for information in the parietal cluster as a whole. These values were subsequently tested across participants against a null hypothesis of zero.

Experiment 2: design. As in Experiment 1, the stimulus set for Experiment 2 consisted of a set of black-and-white pictures of natural scenes $(n=384)$. However, the set of images used in Experiment 2 only partially overlapped with the set of images used in Experiment 1. This is the case because, in Experiment 1, scenes contained examples of either 3 or 4 of the possible target categories. In Experiment 2, scenes contained examples of either 2 or 3 of the categories. Four groups of scenes $(n=48)$ each contained all possible combinations of three categories. Six more groups of scenes contained all possible combinations of two categories. Of these six groups, we had trouble finding life-like examples of car/tree and people/building combinations in the absence of the other categories. As a result, these groups of images contained only 24 examples; all other groups contained 32 images. Scenes were masked using the same images as used in Experiment 1.

Experiment 2 took place in a quiet room and was comprised of 48 blocks of 16 trials. Each block began with a text cue indicating both the target category for the coming block and the pay scheme. This means that, in contrast to the design of Experiment 1, the association of outcome to object categories could be counterbalanced within participants in Experiment 2. For each participant, two object categories (cars and trees) were paired with each of the possible outcomes in an equal number of experimental blocks, with the order of pairing counterbalanced across participants. Two other target categories (people and buildings) were consistently associated with neutral feedback. Blocks were organized in pairs, such that blocks in which participants searched for one of the two outcome-varying categories (cars or trees) were always followed by blocks where they searched for one of the two always-neutral categories (people or buildings). In these neutral-target test blocks, examples of the target category from the immediately preceding block could appear as task-irrelevant distractors.

All blocks contained 8 target-present and 8 target-absent trials. In target-present trials, in addition to the target, the scenes contained an example of 1 of the 3 nontarget categories in half of the trials and examples from 2 of the 3 nontarget categories in the remainder. In targetabsent trials, the scenes contained examples of 2 of the 3 nontarget categories in half of the trials and examples from all 3 nontarget categories in the remainder. This was manipulated such that examples of each nontarget category were present and absent an equal number of times in both target-present and target-absent conditions, allowing us to determine how the presence of each distractor type impacted performance in the neutral test blocks. The trial sequence in Experiment 2 was as illustrated in Figure $2 A$ but with different latencies. Fixation $(833 \mathrm{~ms})$ was followed by presentation of the scene $(40 \mathrm{~ms})$, a mask $(260 \mathrm{~ms})$, the reappearance of fixation $(300 \mathrm{~ms})$, and feedback $(533 \mathrm{~ms})$.

For 54 participants in Experiment 2, the payoff scheme was identical to that used in Experiment 1. For 47 participants, detection of rewardassociated targets garnered 100 points and failure to detect resulted in 0 points, detection of neutral targets resulted in 1 point and failure to detect resulted in the loss of 1 point, and detection of loss-associated targets resulted in 0 points and failure to detect resulted in the loss of 100 points. Payoff for target-absent trials remained as in Experiment 1. Statistical analysis demonstrated no difference in performance across these groups (for all effects involving this difference, $p>0.211$ ). Participants were paid based on the number of points they accumulated during the experiment ( $€ 0.0011$ /point), and each received between $€ 7$ and $€ 12$.

Experiment 2: analysis. Blocks with varying-outcome targets (cars and trees) were analyzed separately from blocks with consistent-outcome targets (people and buildings), with the former focusing on variance as a function of target association (i.e., accuracy for reward- vs loss-associated targets) and the latter focusing on variance as a function of distractor 
A Experiment 1: Visual search in natural scenes

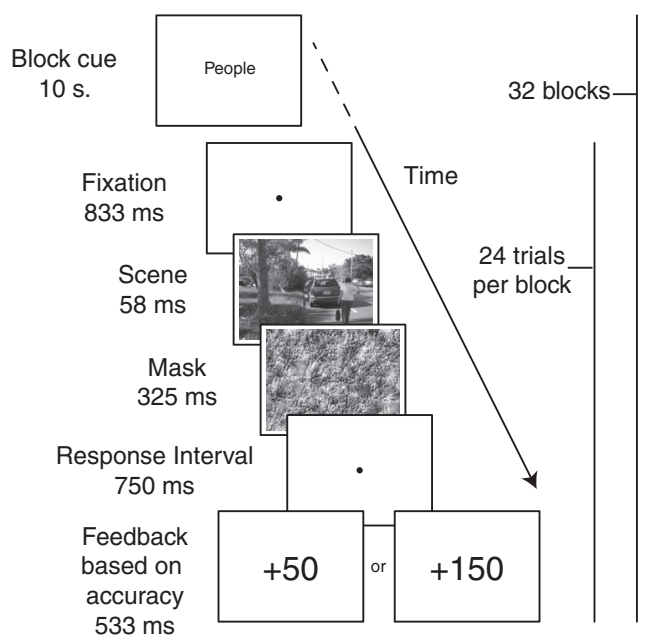

C Correlate Scene-elicited OSC Patterns To Patterns from Category Localizer

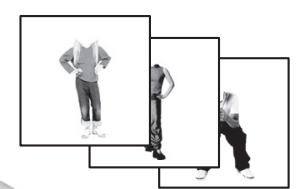

People, Cars, Trees, and Buildings

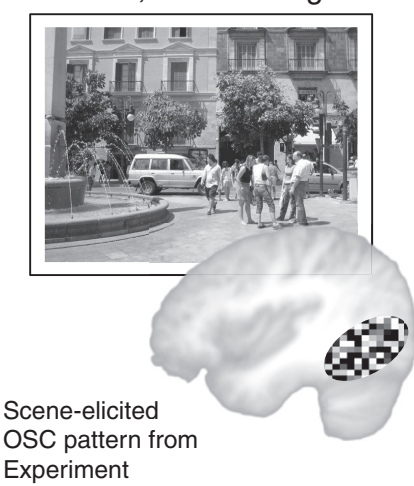

Experiment

\section{B Feedback Schedule}
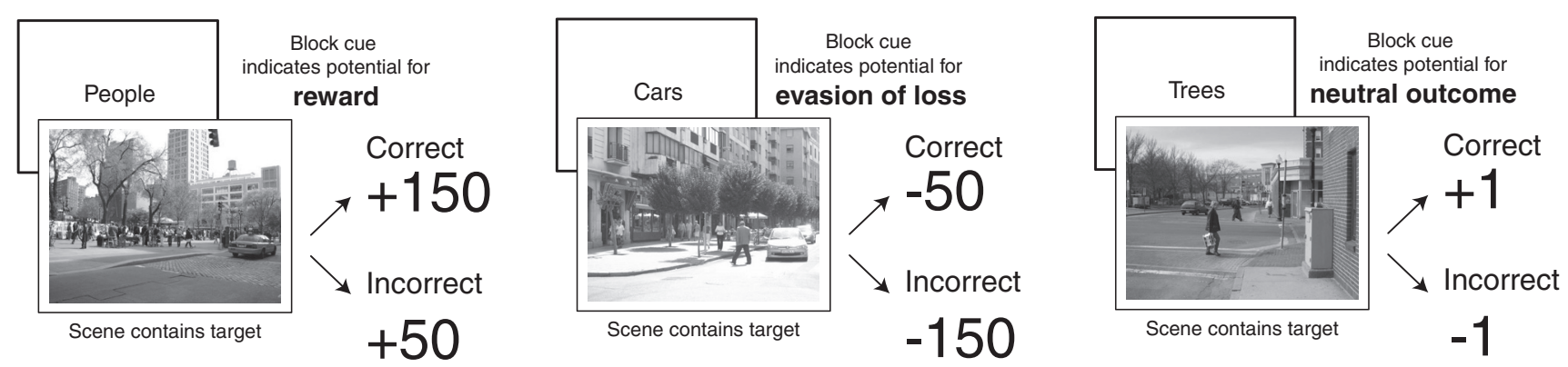

Figure 2. A, Schematic illustration of the trial sequence. Participants reported the presence of examples of the cued category in briefly presented scenes. Of four possible target categories: one was associated with reward, one to loss, and two to neutral outcome. Image and font sizes are not to scale, and the block cue here indicates only the target category for the coming trials, whereas in the experiment itself the total number of points earned to that point in the experiment was also presented. $\boldsymbol{B}$, Feedback schedule. The association of category to outcome in the actual experiment was counterbalanced across participants. Feedback indicated here was for target-present trials. Correct performance in target-absent trials garnered a single point in neutral blocks and 0 points in reward and loss blocks. Incorrect performance in target-absent trials resulted in the loss of a single point in neutral blocks and the loss of 50 points in reward and loss blocks. C, Analytic approach. Scene-evoked activity patterns in OSC were correlated with benchmark patterns. High correlation indicates increased information for that category in visual cortex during scene perception.

association (i.e., accuracy for neutral targets in the presence of distractors that served as reward-, neutral- or loss-associated targets in the preceding block).

Behavioral results from Experiment 2 were analyzed using the same regression approach used in Experiment 1. Detection accuracy was identified for conditions where the target was associated with reward, loss, or neutral outcome, and also for conditions where the target was associated with neutral outcome, but the distractor was associated with reward, loss, or neutral outcome. These six values were regressed to predictors representing the utility and valence models for each participant, and the resulting $\beta$ coefficients were tested against a null hypothesis of zero.

Statistics. All statistics reported in the paper are a product of permutation analysis with two exceptions: the GLM models used to generate parametric fMRI maps and the whole-brain correlation analysis. For permutation tests against the null hypothesis of zero, data-driven distributions were generated by randomly sampling from the relevant dataset $10^{6}$ times with replacement. The likelihood of observed data given the null was calculated compared with these distributions. Tests of multifactor results were conducted by randomly relabeling conditional data over $10^{4}$ iterations to create a data-driven distribution of $F$ values for each main effect and interaction. The likelihood of observed $F$ values given the null was calculated compared with these distributions. Tests of correlation were conducted using a Studentized bootstrap analysis (with $10^{4}$ iterations in the outer loop and 100 permutations in the inner loop).

\section{Results}

The impact of reward and loss on stimuli representations in OSC and behavior

Our first aim was to determine whether the association of reward or loss impacted the visual representation of experimental stimuli in a manner predicted by the utility or valence models. We began by analyzing fMRI results from Experiment 1, deriving measures of category information from voxelwise patterns in object selective visual cortex (OSC). To do this, we first isolated OSC by comparing cortical responses to objects versus scrambled versions of these objects. We then correlated voxelwise patterns evoked in this area during scene viewing with benchmark patterns identified in a separate localizer experiment (in which participants viewed isolated examples of our relevant object categories; Fig. 2C). The degree to which the scene-evoked OSC pattern matched each of the individual category benchmarks provided a measure of the strength with which each of these categories was represented in ventral visual cortex (Peelen et al., 2009; Seidl et al., 2012).

As illustrated in Figure 3, category information was greater for targets than distractors, even though targets were only present in 
A

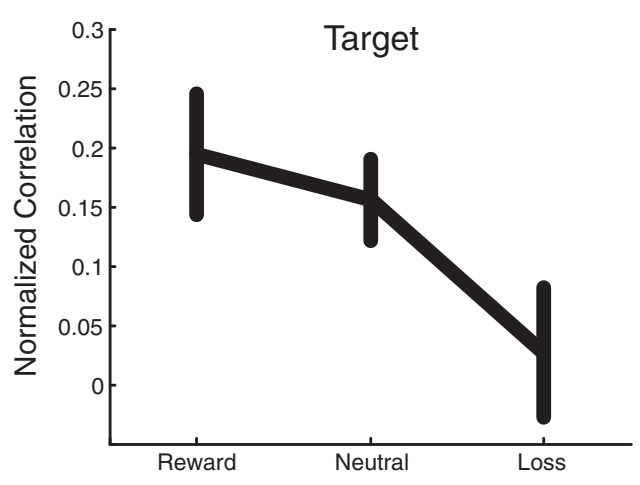

C

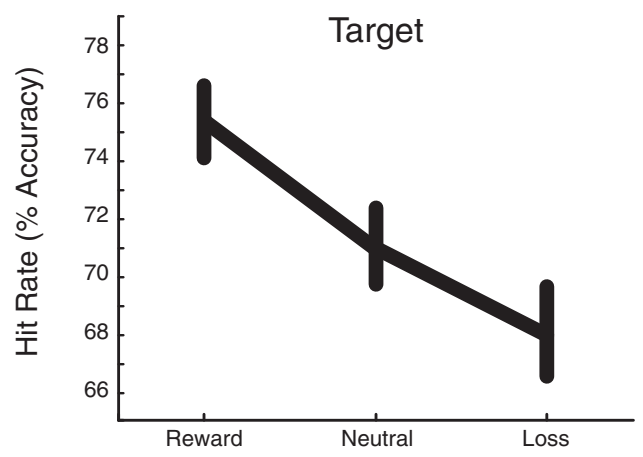

B
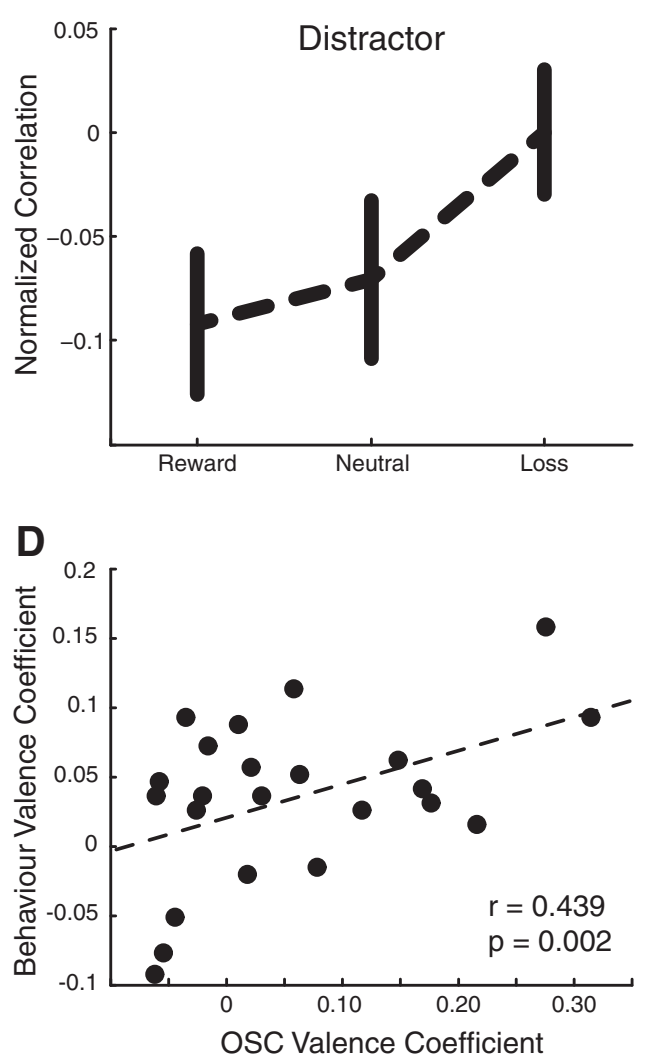

Figure 3. Results from Experiment 1. A, In line with the valence model, reward-associated targets are better represented in OSC than loss-associated targets. $\boldsymbol{B}$, During search for neutral targets, OSC carried less information about reward-associated distractors than loss-associated distractors, indicative of attentional suppression. Our normalization procedure causes these values to be represented on an interval scale with an uninformative zero point. As such, negative values do not imply the presence of information in the form of negative correlation (for details, see Materials and Methods). C, Accuracy in detecting the target when it was present in the scene. $\boldsymbol{D}$, The relationship between coefficients from a fit of the valence model to 0SC category information, as illustrated in $\boldsymbol{A}, \boldsymbol{B}$, and coefficients from a fit of the valence model to hit rate data, as illustrated in C. Error bars indicate within-participant SE (Cousineau, 2005).

half of the trials whereas all distractors were present in every trial. The effect is evident in a scale shift in category information in comparison with Figure $3 A, B$. This strong modulation by topdown set replicates prior studies using this technique to investigate naturalistic visual search (Peelen et al., 2009; Seidl et al., 2012; Hickey and Peelen, 2015). Variance in category information as a function of outcome association closely followed the valence model: OSC carried more information about rewardassociated targets than loss-associated targets (Fig. $3 A$ ), but this pattern reversed for distractors, with OSC carrying less information about reward-associated distractors than loss-associated distractors (Fig. 3B).

Behavioral analysis of Experiment 1 was focused on target detection accuracy when the target was present in the scene (i.e., hit rate) as an analog of the target OSC information effect described above. As illustrated in Figure $3 C$, hit rate paralleled the pattern observed in target category information, and thus also closely followed predictions from the valence model. Importantly, no corresponding effect was observed in accuracy when targets were absent from the scene (i.e., correct rejects; 69\%, 72\%, and $71 \%$ accuracy, respectively), suggesting that outcome association impacted the ability to detect the target rather than increasing the overall propensity that the target would be reported as present.

To statistically assess the ability of the valence and utility models to predict our results, we conducted linear regression analyses. In analysis of imaging data, these had three factors: the first pre- dicted that category information for task relevant stimuli would be greater than for distractors (Peelen et al., 2009; Seidl et al., 2012; Hickey and Peelen, 2015), the second that results would follow the utility model (as illustrated in Fig. $1 A, B$ ), and the third that results would follow the valence model (as illustrated in Fig. $1 C, D)$. This garnered 3 regression coefficients for each participant reflecting the degree to which the data varied according to each of these hypotheses. The coefficients corresponding to the task-relevance model were significantly positive $\left(p<10^{-6}\right)$, as were the coefficients corresponding to the valence model $(p=$ 0.003), demonstrating that these factors reliably predicted our results. However, coefficients for the utility model did not differ from zero ( $p=0.789$ ). Follow-up contrasts revealed that OSC carried more information about reward-associated targets than loss-associated targets $(p=0.002)$ but less information about reward-associated distractors than loss-associated distractors $(p=0.018)$. In OSC, the valence model better characterized the pattern of category information than did the utility model.

Because all distractor types were present in every scene, analysis of behavioral data from Experiment 1 was limited to the effect of outcome association with target stimuli. Regression analysis therefore had two factors: the first predicted that hit rate would follow the utility model (as illustrated in Fig. 1A) and the second that it would follow the valence model (as illustrated in Fig. 1C). Coefficients corresponding to the valence model were significantly positive ( $p=0.002$ ), but coefficients for the utility model did not differ from zero $(p=0.360)$. Follow-up analysis demon- 
strated that the effect of target-outcome association on hit rate reliably differed from its effect on correct rejects (interaction, $p=0.017)$. As was the case in analysis of OSC category information, the valence model better characterized detection accuracy than did the utility model.

To gain further perspective on the relationship between brain activity and behavior, we examined individual differences in how the valence and utility models fit OSC category information and behavior. Our expectation was that those participants showing a strong fit of the valence model in brain activity should also show a strong fit of the model in behavior. To test this hypothesis, we Pearson correlated the valence model coefficients derived from brain data (Fig. $3 A, B$ ) with the valence model coefficients derived from hit rate data (Fig. $3 C$ ). As illustrated in Figure $3 D$, a reliable relationship emerged $(r=0.439, p=0.002)$. No corresponding relationship was evident when utility model coefficients for OSC category information and behavior were correlated $(r=0.075$, $p=0.351)$. Expression of the valence pattern in brain activity therefore predicted a valence pattern in behavior, demonstrating a close relationship between brain activity and behavior.

\section{Midbrain activity as a predictor of representational quality in OSC}

Our study was motivated in part by theories of dopamine's role in attentional selection and approach behavior (Berridge and Robinson, 1998; Ikemoto and Panksepp, 1999), alongside results relating reward's impact on selection to activity in the dopaminergic midbrain (Hickey and Peelen, 2015, 2017) and the release of dopamine in the striatum (Anderson et al., 2016). To test the relationship between midbrain activity and OSC representations in the current data, we used an MRI atlas to define an ROI describing the bilateral SN (Fig. 4A). The dorsal aspect of this SN ROI, encompassing pars compacta and including voxels in the ventral tegmental area (VTA), contains dopaminergic neurons that project to the striatum and frontal cortex (Williams and Goldman-Rakic, 1993; Haber et al., 2000). As a functionally distinct proximal control area, we additionally isolated the bilateral red nuclei, which are located slightly medial and rostral to the SN but do not contain dopaminergic cells and have different connectivity than the $\mathrm{SN}$ (Fig. 4A) (Nioche et al., 2009).

Our expectation was that participants showing greater responsivity in $\mathrm{SN}$ to
A
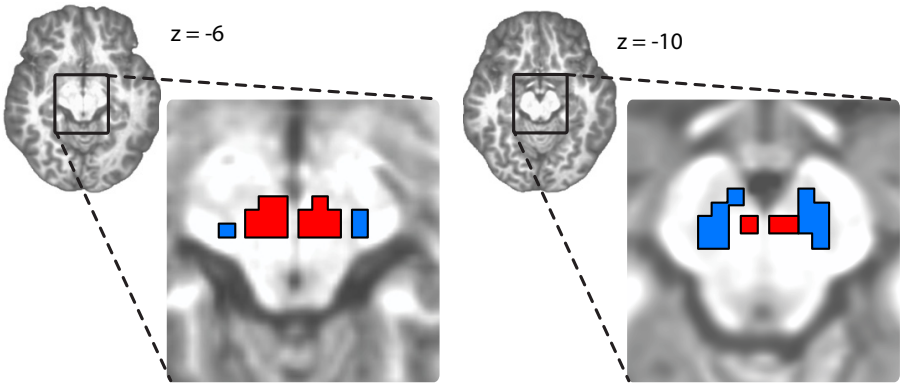

Substantia Nigra / VTA $\square$ Red Nucleus

B
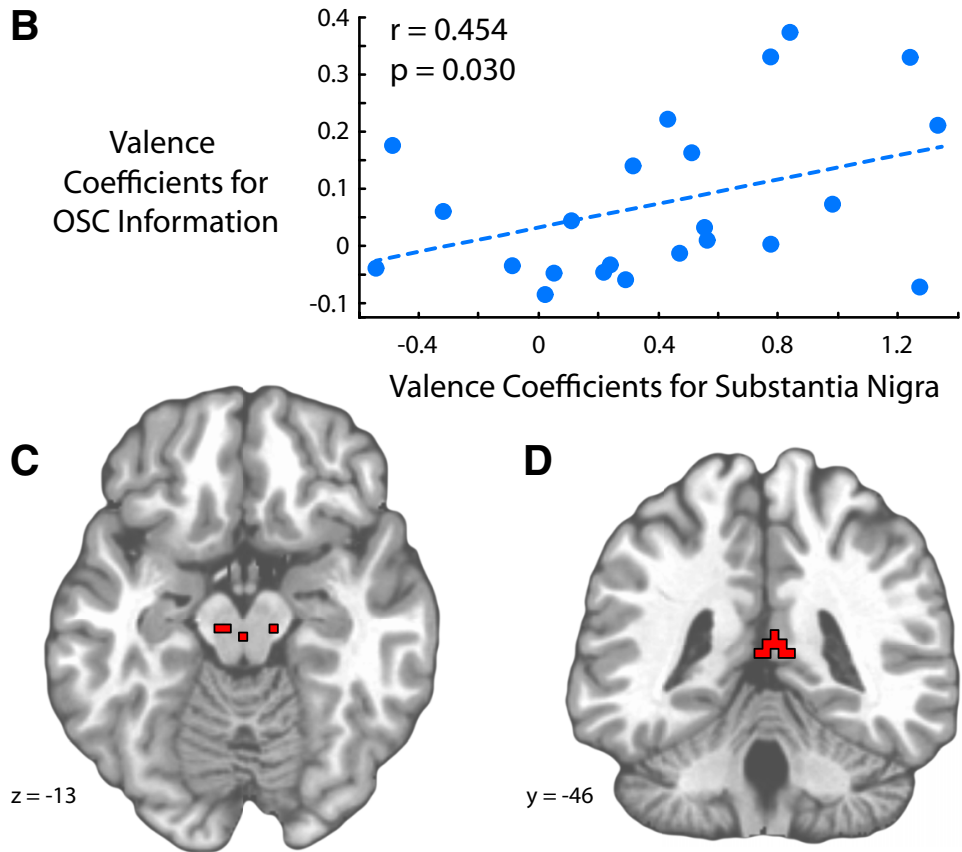

D

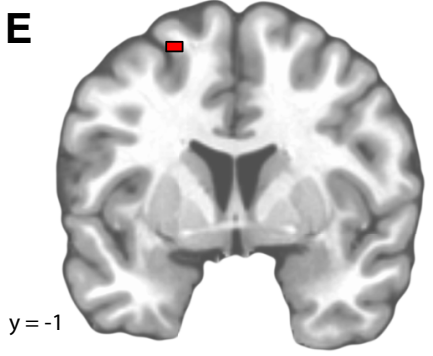

F
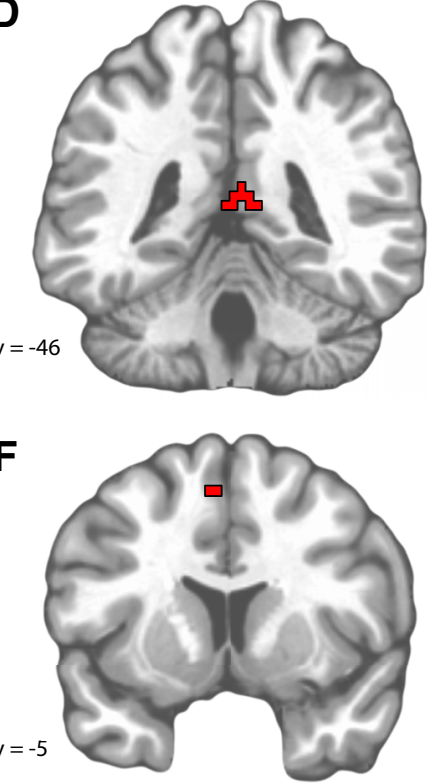

G

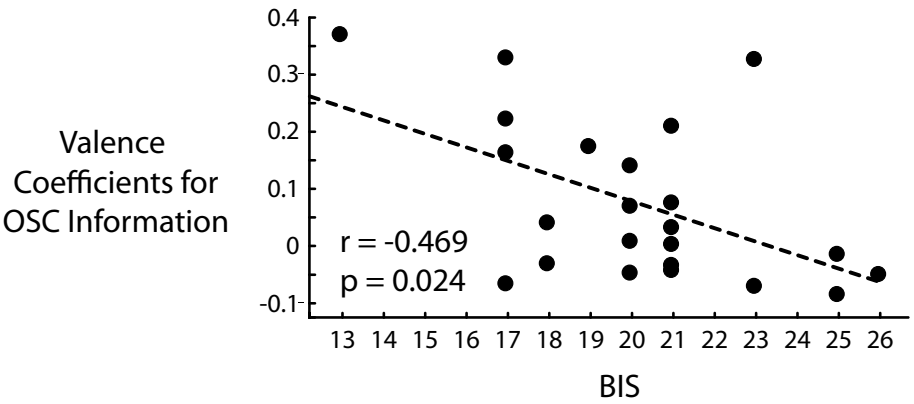

Figure 4. $\quad \boldsymbol{A}$, Anatomically defined ROIs characterizing the $S N$ and red nucleus. $\boldsymbol{B}$, Relationship between interindividual variance in the valence coefficient of the SN ROI and the valence coefficient of category information in OSC. $\boldsymbol{C}$, Midbrain voxels identified in whole-brain correlational analysis. The single $3 \mathrm{~mm}$ voxel on the midline actually begins one slice inferior to the illustrated horizontal section (beginning at $z=-14$ ). $\boldsymbol{D}-\boldsymbol{F}, 0$ ther clusters identified in whole-brain correlational analysis. $\mathbf{G}$, Relationship between interindividual variance in BIS and the valence coefficient of category information in OSC. 

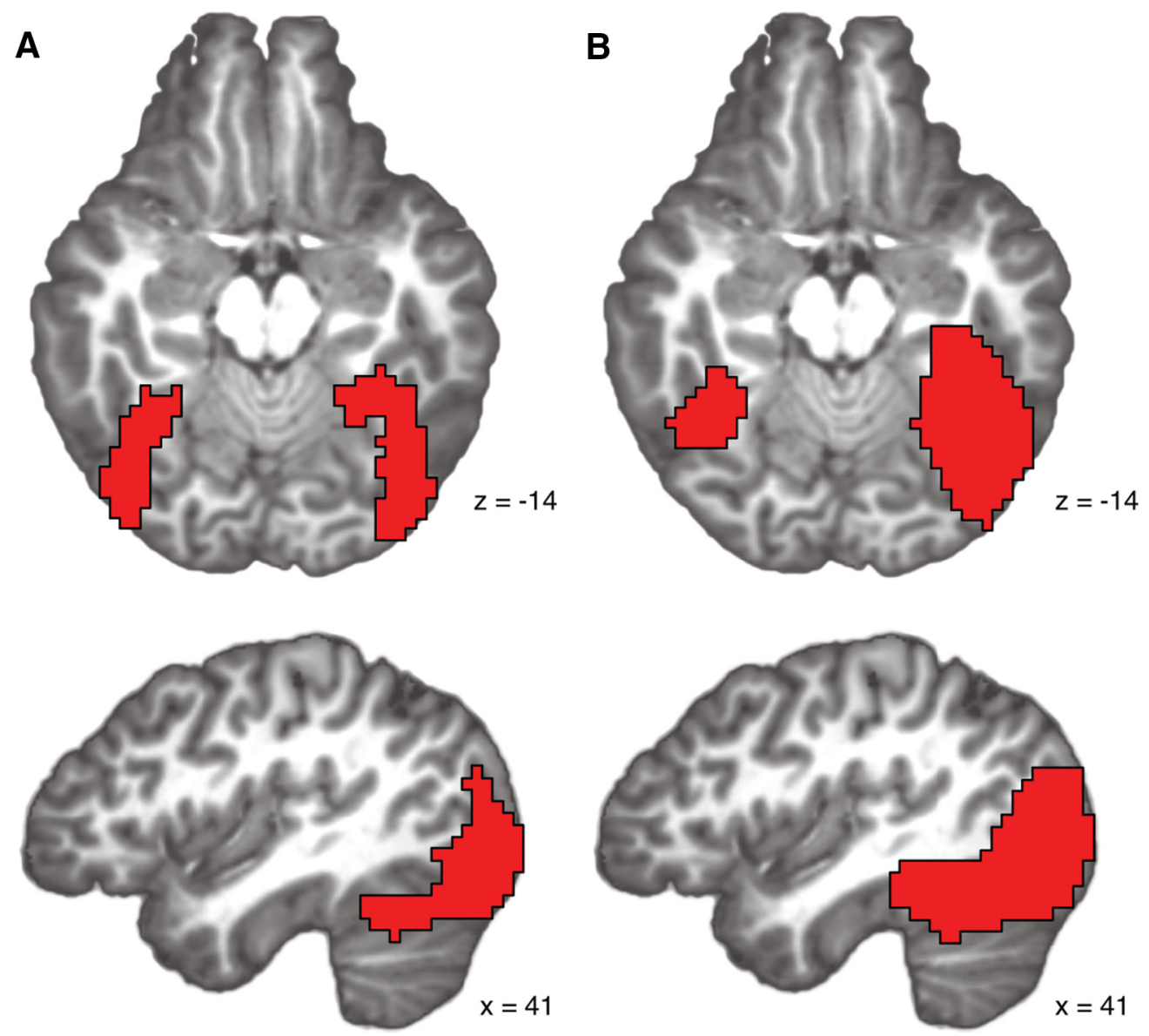

Figure 5. $A, 0 S C$ as defined in the OSC localizer. Voxels identified here were present in the OSC of 16 or more of the 23 participants in Experiment 1. B, Results from the searchlight contrast of information for targets versus distractors. Voxels identified here constitute the center of spheres that carried more information for targets than distractors at $p<0.001$ with a cluster threshold of 50 voxels.

reward- versus loss-associated targets would also show increased category information for these stimuli in OSC. To test this, we conducted a univariate regression analysis of voxel activation in the $\mathrm{SN}$ and red nuclei ROIs with predictors representing the utility and valence models. The parameter values identified in this univariate analysis of SN and red nucleus were subsequently correlated with valence model coefficients from multivariate analysis of target information in OSC. As illustrated in Figure $4 B$, participants showing a valence pattern in the $\mathrm{SN}$ also showed this pattern in OSC category information $(r=0.387, p=0.030)$. This relationship was not evident for the red nucleus $(r=0.054, p=$ 0.397 ), and correlation of utility model coefficients for OSC information and SN activity identified no relationship $(r=-0.019$, $p=0.538$ ). These findings demonstrate that, when the valence pattern discretely expressed in the $\mathrm{SN}$, it also expressed in OSC category information.

We conducted an additional whole-brain analysis using this analytic approach. Here, coefficient values were extracted for each participant based on univariate activation of each voxel in brain space and subsequently correlated with OSC category information coefficients. After FDR correction for multiple comparisons (Benjamini and Hochberg, 1995), this analysis revealed a set of small voxel clusters where a good fit of the valence model to univariate activity predicted a good fit of the valence model to OSC category information. Supporting the ROI analysis detailed above, a set of voxels was identified in the midbrain (Fig. 4C). These were located bilaterally in the SN (left: 2 voxels; peak: -9 ,
$-25,-11$; right: 1 voxel; $12,-25,-11)$ and in the vicinity of the ventral tegmental area ( 1 voxel; $0,-29,-15)$. Other clusters emerged in the posterior cingulate cortex (Fig. 4D; 15 voxels; peak: $-9,-46,16$ ), left superior frontal gyrus (Fig. $4 E ; 10$ voxels; peak: $-21,-1,65)$, and left medial frontal gyrus/dorsal anterior cingulate (Fig. 4F; 3 voxels; peak: $-6,5,58$ ). The superior frontal, anterior cingulate, and midbrain clusters are similar to those that we have identified using similar methodology in earlier investigation of incentive salience (Hickey and Peelen, 2015).

\section{Beyond OSC: whole-brain analysis of category information}

In the imaging analyses described to this point, we have measured representational quality based on consideration of voxel patterns in OSC, which constitutes a large portion of ventral visual cortex spanning the occipital and temporal lobes (Fig. 5A). However, other brain areas are known to also carry multivoxel information about naturalistic visual stimuli and their task relevance (Konen and Kastner, 2008; Jeong and $\mathrm{Xu}, 2016$ ). To test the ability of the valence and utility models to describe variance in the quality of object representation outside OSC, we conducted a whole-brain searchlight analysis of the fMRI data (Kriegeskorte et al., 2006).

This began with the identification of brain areas that carried more information about the target of visual search than about task-irrelevant distractors, such that category information in these areas could be subsequently examined for the effect of out- 
come association. We defined spheres around each voxel in the brain, testing whether the pattern of voxelwise activity in each area correlated with corresponding values from benchmark data generated in our localizer task. This identified three areas that carried more information about targets than distractors. Two of these were in the bilateral ventral visual cortex, approximately equivalent in location and size to OSC as discussed above (Fig. 5). Consistent with other recent work (Bettencourt and $\mathrm{Xu}, 2016$; Jeong and $\mathrm{Xu}, 2016)$ a third cluster emerged in right posterior parietal cortex, notably containing aspects of the intraparietal sulcus and superior parietal lobule (Fig. 6A) (compare Peck et al., 2009).

We constrained subsequent analysis to this parietal cluster, extracting category information for targets and distractors as a function of outcome association. In a reversal of the pattern we saw in OSC, mean category information here paralleled the utility rather than valence model: category information was equal for reward- and loss-associated targets, in both cases larger than for neutral targets (Fig. 6B), but this reversed in analysis of distractor information, with parietal cortex carrying less information about both reward- and loss-associated distractors than neutral distractors (Fig. 6C).

We once again used linear regression to statistically assess these results. For each participant and searchlight sphere, we modeled category information with predictors for task relevance, the utility model, and the valence model. We subsequently generated a set of mean coefficients for each participant that described the degree to which information in the parietal cluster was characterized by our models. The parietal cluster was defined based on its sensitivity to task relevance, so coefficients corresponding to the task relevance predictor were unsurprisingly positive $\left(p<10^{-6}\right)$. Utility model coefficients were also positive $(p=0.002)$, demonstrating the explanatory power of this model, but valence model coefficients did not differ from zero $(p=$ 0.511).

Follow-up contrasts revealed that parietal cortex trended toward carrying more information about a reward-associated target than a neutral target $(p=0.108)$ and about a loss-associated target than a neutral target $(p=0.143)$. Equivalent analyses of distractor information garnered more reliable effects, with less information about a reward-associated distractor than a neutral distractor $(p=0.015)$ and less information about a lossassociated distractor than a neutral distractor $(p=0.010)$.

Personality as a predictor of representational quality in OSC We have previously found that selective biases to rewardassociated stimuli, as evident in behavior (Hickey et al., 2015) and OSC category information (Hickey and Peelen, 2015, 2017), can vary across individuals as a function of personality traits related to reward sensitivity. With this in mind, we approached the current study with the idea that personality traits related to the valuation of reward and loss might predict the degree to which representations in OSC followed the utility and valence models, and participants in Experiment 1 completed a native-language version of the behavioral inhibition score (BIS)/behavioral activation score (BAS) personality scale (Carver and White, 1994; Leone et al., 2002) immediately after the scanning session. This scale generates two primary values: BIS, reflecting sensitivity to punishment; and BAS, reflecting sensitivity to reward.

We correlated BIS and BAS scores across participants with OSC coefficients for the valence model. This identified a negative correlation between BIS and OSC valence coefficients (Fig. 4G; $r=-0.501, p=0.021)$, and a nonsignificant relationship with BAS $(r=-0.122, p=0.294)$. It is important to point out, however, that the significance of the relationship between BIS and OSC valence coefficients did not sustain when the single participant with a BIS score of 13 was removed from analysis $(r=-0.370, p=$ $0.056)$. This finding suggests that the overall propensity for stronger OSC representation of reward-associated rather than lossassociated targets (and vice versa for distractors) may be reduced in participants with high sensitivity to negatively valenced outcome. But the pattern should be interpreted with caution given the marginal statistics.

\section{The impact on behavior of reward and loss associations to distractors}

Experiment 1 identified a difference in detection accuracy for reward- and loss-associated targets. However, because all distractor categories were present in every trial, there was no opportunity to determine how distractors impacted task performance. We accordingly conducted a second behavioral experiment to further examine the effect of reward- and loss-associated distractors on search behavior. In Experiment 2, each block began with a cue indicating both the target category and pay scheme for the 
A

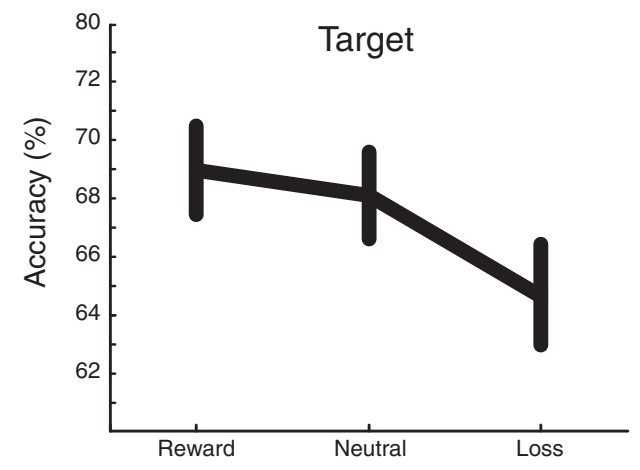

B

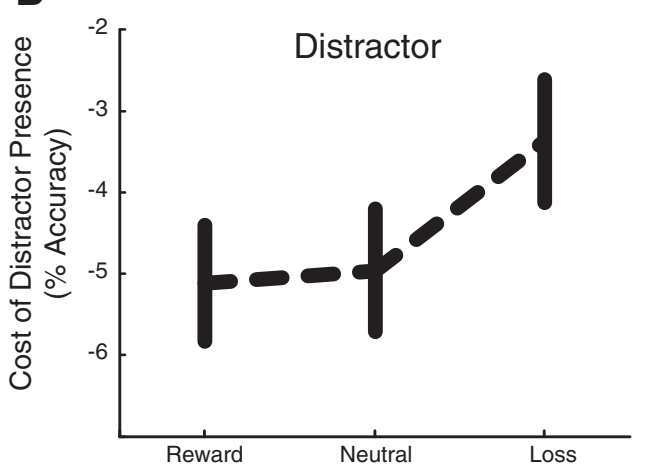

Figure 7. Results from Experiment 2. $\boldsymbol{A}$, In line with the valence model, accuracy is better for reward-associated targets than loss-associated targets. $\boldsymbol{B}$, Search for a neutral target is more strongly disrupted by a reward- versus loss-associated distractor.

coming trials. Blocks were organized in pairs such that blocks involving reward-associated, loss-associated, or neutral targets were consistently followed by a block where the target was neutral. In these test blocks, examples of the target category from the immediately preceding block could appear as task-irrelevant distractors, allowing us to determine how distractors recently associated with reward or loss impacted the search for the neutral target.

As illustrated in Figure 7A, $B$, response accuracy in Experiment 2 paralleled the pattern of OSC information observed in Experiment 1, thus also closely following predictions from the valence model. Once again, per-participant regression analyses were used to assess the predictive power of the models. Coefficients corresponding to the valence model were positive $\left(p<10^{-4}\right)$, but coefficients corresponding to the utility model were negative $(p=0.036)$, reflecting an inverse relationship between the model and experimental results (and thus a very bad fit). Follow-up contrasts demonstrated that detection of reward-associated targets was better than of lossassociated targets $\left(p<10^{-4}\right)$, but that detection of neutral targets was degraded more by a reward-associated distractor than by a loss-associated distractor $(p=0.045)$.

More detailed analyses of behavior are presented in Figure 8. Our prior work with reward-associated stimuli has demonstrated that nonstrategic reward-driven prioritization expresses in accuracy, response latency, or both measures, depending on task confines (Hickey et al., 2010; Hickey and Peelen, 2015) but is short-lived and can be followed by attentional suppression (Hickey and van Zoest, 2012; Hickey and Peelen, 2015). With this in mind, participants in Experiment 2 were required to respond within $600 \mathrm{~ms}$ of stimulus onset. This created two types of task error: explicit errors, when participants incorrectly reported the presence or absence of the target; and time-out errors, where they failed to respond in the critical interval. Separating these, we found that participants made fewer explicit errors when scenes contained a reward-associated rather than loss-associated target (Fig. 8A). This did not reflect a response bias: if participants were simply more likely in the reward condition to report the target-present, they would have made many errors when the target was indeed absent. But participants were also nominally more accurate in reporting the absence of a reward-associated target (Fig. $8 B$ ). Accordingly, signal detection analysis shows a greater $d^{\prime}$ in the reward condition than in the loss condition (Fig. 8C) with no concomitant change in criterion (Fig. $8 D$ ). When participants responded within the time limit, they were slower to do so for a loss-associated target rather than a reward-associated target (Fig. $8 E$ ), and the cost in accuracy created by the presence of a reward-associated distractor (Fig. 7B) was not a product of speed-accuracy tradeoff, as target responses were not any faster under this circumstance (Fig. $8 F$ ). Consistent with the reaction time slowing, participants were less likely to respond within the time limit in this loss condition (Fig. 8G). This effect on time-out errors was significantly greater when the target was present than when it was absent (Fig. $8 \mathrm{H}$; interaction, $p=0.004$ ), suggesting that this reflects a difficulty in target processing rather than a more general slowing of response in the loss condition.

\section{Discussion}

Economically rational preferences are defined by their logical consistency: they remain the same across irrelevant changes to circumstance and context (Arrow, 1982; Tversky and Kahneman, 1989; Glimcher, 2010). Here we ask whether selective bias for visual stimuli associated with economic benefit meets this criterion. We designed a naturalistic visual search task in which participants earned points with cash value by detecting examples of object categories in images of scenes. Detection of reward-associated targets resulted in a gain of 150 points with misses garnering 50 points, whereas detection of loss-associated targets resulted in the forfeiture of 50 points but saved participants from the greater loss of 150 points. Correct detection of both reward- and loss-associated targets therefore had a benefit of 100 points, but this was couched in the irrelevant context of absolute gain or loss.

If the ability of reward-associated stimuli to draw attention reflects a normative visual preference for objects that can be used to maximize utility, this design should cause reward-associated and loss-associated stimuli to draw attention equally well. But results from analysis of category information in OSC (Experiment 1) and task performance (Experiment 2) show no hint of this pattern. Consistent with prior work, reward-associated stimuli were easy to detect and well represented in OSC (Hickey and Peelen, 2015). But loss-associated stimuli were not prioritized in the same way. Selective bias for rewardassociated object categories in natural scenes thus appears to reflect an irrational visual preference for objects associated with positive-valence outcome, not a rational sensitivity for stimuli that maximize utility.

This bias in visual representation parallels known effects in economic decision-making. Tversky and Kahneman (1981) 
famously showed that the perceived utility of a choice is sensitive to the context in which the options are presented, with change in this "choice frame" strongly impacting decision-making and behavior. As a result, people are more likely to choose an option presented in a positive frame than a negative frame, even when the value of outcome does not change. This has been interpreted as reflecting the use of simplifying heuristics, such as reliance on emotional content, that forego effortful reasoning and valuation of options (Loewenstein et al., 2001).

By framing the benefit of correct performance in the irrelevant context of absolute loss, we may therefore have caused participants to devalue this outcome altogether. However, results from wholebrain searchlight analysis of our fMRI data argue against this possibility. This identified rational, utility-maximizing stimuli representations in the intraparietal sulcus and superior gyrus of the parietal lobe. This is broadly consistent with existing neurophysiological and fMRI studies suggesting that IPS may code the true value of environmental stimuli (Leathers and Olson, 2012; e.g., Kahnt et al., 2014). But in the current results, these utility-driven representations did not influence encoding in OSC and did not drive task performance.

One possibility is that development of these parietal representations was slower than the impact of valence and thus could not influence the representation of stimuli before the preparation and execution of response. The idea that valence may have an earlier impact on attentional control than does strategy is consistent with existing work using time-sensitive measures (Hickey et al., 2010; Hickey and van Zoest, 2012; Buschschulte et al., 2014) and in line with the broad idea that prioritization of reward-related stimuli may reflect longterm plasticity in visual cortex triggered by neuromodulatory signals (Roelfsema et al., 2010; Hickey and Peelen, 2015, 2017). Our results further support the idea that OSC representations may be influenced by neuromodulatory systems by showing that these representations vary as a function of signaling in midbrain nuclei known to contain dopaminergic cells.

At first glance, our results appear at odds with a literature demonstrating attentional bias toward negatively valenced stimuli associated with threat or pain (Pessoa and Adolphs, 2010; Tami-

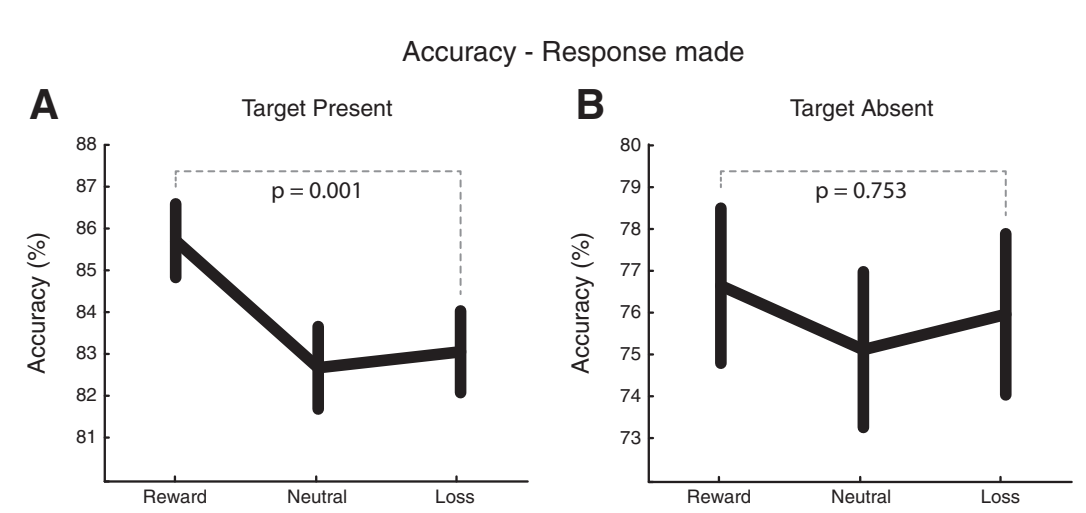

Signal Detection - Response made
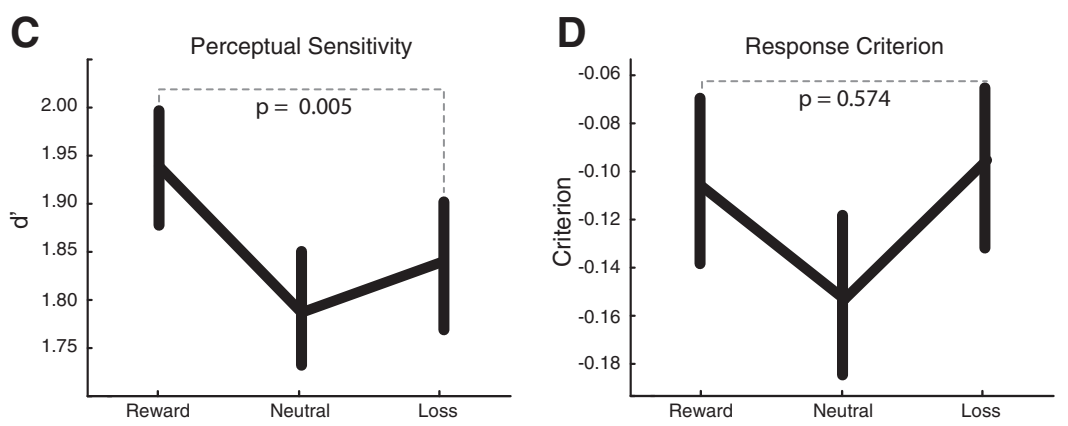

Reaction Times - Response made
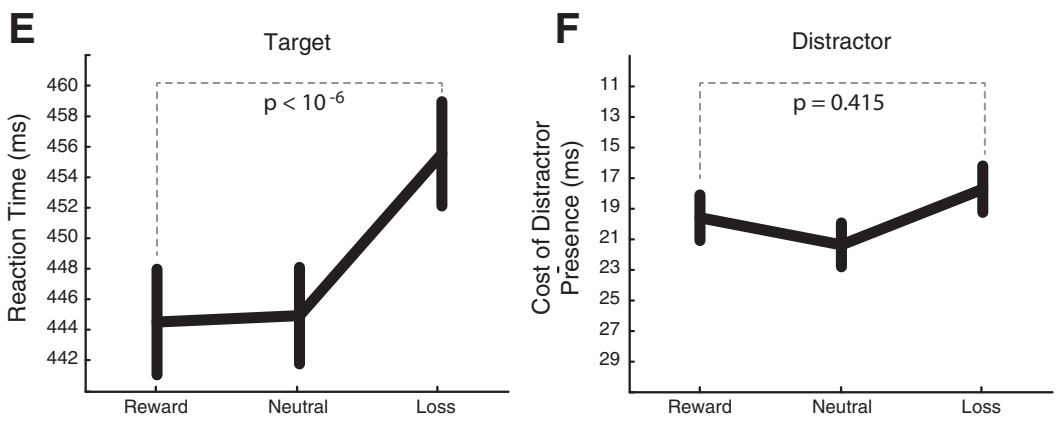

Time-outs - No response made
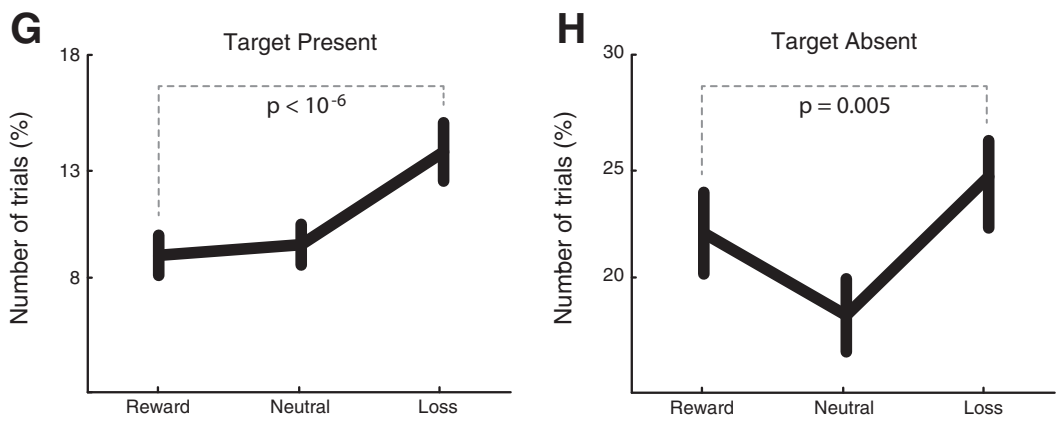

Figure 8. Further analysis of results from Experiment 2. A, Explicit accuracy in target-present trials as observed when a response was made within the time limit. $\boldsymbol{B}$, Explicit accuracy in target-absent trials. $\boldsymbol{C}$, Perceptual sensitivity for targets. $\boldsymbol{D}$, Response criterion. Participants tended to report the target as present, resulting in a negative criterion, but this did not differ across reward and loss conditions. $\boldsymbol{E}$, Reaction times for target-present and target-absent trials as a function of target association. $\boldsymbol{F}$, Reaction times for target-present and target-absent trials where the target had neutral association, as a function of whether a reward-, loss-, or neutral-associated distractor was present in the scene. G, Number of time-out trials when target was present, as proportion of total per condition. $\boldsymbol{H}$, Number of time-out trials when target was absent. Error bars indicate within-participant SE (Cousineau, 2005). 
etto and de Gelder, 2010). However, this bias is thought to be unique, perhaps relying on a distinct subcortical route for visual information (Tamietto and de Gelder, 2010). There are relatively few studies investigating attentional bias to stimuli associated with economic loss, and these have garnered mixed results (Schacht et al., 2012; Wang et al., 2013; Wentura et al., 2014). Studies using disgusting images, however, show that these are rapidly suppressed and subsequently ignored (e.g., Zimmer et al., 2015). The emotion evoked by stimuli associated with small, unavoidable economic loss strikes as potentially more similar to disgust than fear, and it is perhaps reasonable that the visual representation of our loss-associated objects is similar to that of stimuli associated with disgust.

In conclusion, we show an economically irrational influence of prior experience on search for naturalistic environmental stimuli and the encoding of these stimuli in ventral visual cortex. Normative models of economic behavior propose that human preferences are consistent and rational, but results from studies of decision-making and behavioral economics show that this is commonly not the case (for review, see Glimcher, 2010). Here we demonstrate that this economic irrationality in human cognition begins as early as during perceptual encoding of our environment.

\section{References}

Anderson BA (2016) The attention habit: how reward learning shapes attentional selection. Ann N Y Acad Sci 1369:24-39. CrossRef Medline

Anderson BA, Laurent PA, Yantis S (2011) Value-driven attentional capture. Proc Natl Acad Sci U S A 108:10367-10371. CrossRef Medline

Anderson BA, Kuwabara H, Wong DF, Gean EG, Rahmim A, Brašić JR, George N, Frolov B, Courtney SM, Yantis S (2016) The role of dopamine in value-based attentional orienting. Curr Biol 26:550-555. CrossRef Medline

Arrow KJ (1982) Risk perception in psychology and economics. Econ Inquiry 20:1-9. CrossRef

Benjamini Y, Hochberg Y (1995) Controlling the false discovery rate: a practical and powerful approach to multiple testing. J R Stat Soc B 57: 289-300.

Berridge KC (1996) Food reward: brain substrates of wanting and liking. Neurosci Biobehav Rev 20:1-25. CrossRef Medline

Berridge KC, Robinson TE (1998) What is the role of dopamine in reward: hedonic impact, reward learning, or incentive salience? Brain Res Rev 28:309-369. CrossRef Medline

Bettencourt KC, Xu Y (2016) Decoding the content of visual short-term memory under distraction in occipital and parietal areas. Nat Neurosci 19:150-157. CrossRef Medline

Buschschulte A, Boehler CN, Strumpf H, Stoppel C, Heinze HJ, Schoenfeld MA, Hopf JM (2014) Reward- and attention-related biasing of sensory selection in visual cortex. J Cogn Neurosci 26:1049-1065. CrossRef Medline

Carver CS, White TL (1994) Behavioral inhibition, behavioral activation, and affective responses to impending reward and punishment: the BIS/ BAS Scales. J Pers Soc Psychol 67:319-333. CrossRef

Cousineau D (2005) Confidence intervals in within-participant designs: $s$ simpler solution to Loftus and Masson's method. Tutorials Quant Methods Psychol 1:42-45. CrossRef

Glimcher PW (2010) Foundations of neuroeconomic analysis. Oxford: Oxford UP.

Gottlieb J (2012) Attention, learning, and the value of information. Neuron 76:281-295. CrossRef Medline

Haber SN, Fudge JL, McFarland NR (2000) Striatonigrostriatal pathways in primates form an ascending spiral from the shell to the dorsolateral striatum. J Neurosci 20:2369-2382. Medline

Haxby JV, Gobbini MI, Furey ML, Ishai A, Schouten JL, Pietrini P (2001) Distributed and overlapping representations of faces and objects in ventral temporal cortex. Science 293:2425-2430. CrossRef Medline

Hickey C, Peelen MV (2015) Neural mechanisms of incentive salience in naturalistic human vision. Neuron 85:512-518. CrossRef Medline

Hickey C, Peelen MV (2017) Reward selectively modulates the lingering neural representation of recently attended objects in natural scenes. J Neurosci 37:7297-7304. CrossRef Medline

Hickey C, van Zoest W (2012) Reward creates oculomotor salience. Curr Biol 22:R219-R220. CrossRef Medline

Hickey C, Chelazzi L, Theeuwes J (2010) Reward changes salience in human vision via the anterior cingulate. J Neurosci 30:11096-11103. CrossRef Medline

Hickey C, Kaiser D, Peelen MV (2015) Reward guides attention to object categories in real-world scenes. J Exp Psychol 144:244-273. CrossRef Medline

Ikemoto S, Panksepp J (1999) The role of nucleus accumbens dopamine in motivated behavior: a unifying interpretation with special reference to reward-seeking. Brain Res Rev 31:6-41. CrossRef Medline

Jeong SK, Xu Y (2016) Behaviorally relevant abstract object identity representation in the human parietal cortex. J Neurosci 36:1607-1619. CrossRef Medline

Kahnt T, Park SQ, Haynes JD, Tobler PN (2014) Disentangling neural representations of value and salience in the human brain. Proc Natl Acad Sci U S A 111:5000-5005. CrossRef Medline

Konen CS, Kastner S (2008) Two hierarchically organized neural systems for object information in human visual cortex. Nat Neurosci 11:224-231. CrossRef Medline

Kriegeskorte N, Goebel R, Bandettini P (2006) Information-based functional brain mapping. Proc Natl Acad Sci U S A 103:3863-3868. CrossRef Medline

Leathers ML, Olson CR (2012) In monkeys making value-based decisions, LIP neurons encode cue salience and not action value. Science 338:132135. CrossRef Medline

Leone L, Pierro A, Mannetti L (2002) Validità della versione Italiana delle scale BIS/BAS di Carver e White 1994: generalizzabilità della struttura e relazioni con costrutti affini. Giornale Italiano Psicologia 29:413-436.

Le Pelley ME, Mitchell CJ, Beesley T, George DN, Wills AJ (2016) Attention and associative learning in humans: an integrative review. Psychol Bull 142:1111-1140. CrossRef Medline

Loewenstein GF, Weber EU, Hsee CK, Welch N (2001) Risk as feelings. Psychol Bull 127:267-286. CrossRef Medline

Maldjian JA, Laurienti PJ, Kraft RA, Burdette JH (2003) An automated method for neuroanatomic and cytoarchitectonic atlas-based interrogation of fMRI data sets. Neuroimage 19:1233-1239. CrossRef Medline

Misaki M, Kim Y, Bandettini PA, Kriegeskorte N (2010) Comparison of multivariate classifiers and response normalizations for pattern-information fMRI. Neuroimage 53:103-118. CrossRef Medline

Nioche C, Cabanis EA, Habas C (2009) Functional connectivity of the human red nucleus in the brain resting state at 3t. AJNR Am J Neuroradiol 30:396-403. CrossRef Medline

Oosterhof NN, Connolly AC, Haxby JV (2016) CoSMoMVPA: multimodal multivariate pattern 94 analysis of neuroimaging data in Matlab/ GNU Octave. Front Neuroinform 10:27. CrossRef Medline

Peck CJ, Jangraw DC, Suzuki M, Efem R, Gottlieb J (2009) Reward modulates attention independently of action value in posterior parietal cortex. J Neurosci 29:11182-11191. CrossRef Medline

Peelen MV, Fei-Fei L, Kastner S (2009) Neural mechanisms of rapid natural scene categorization in human visual cortex. Nature 460:94-97. CrossRef Medline

Pessoa L, Adolphs R (2010) Emotion processing and the amygdala: from a 'low road' to 'many roads' of evaluating biological significance. Nat Rev Neurosci 11:773-783. CrossRef Medline

Robinson TE, Berridge KC (1993) The neural basis of drug craving: an incentive-sensitization theory of addiction. Brain Res Rev 18:247-291. CrossRef Medline

Roelfsema PR, van Ooyen A (2005) Attention-gated reinforcement learning of internal representations for classification. Neural Comput 17:21762214. CrossRef Medline

Roelfsema PR, van Ooyen A, Watanabe T (2010) Perceptual learning rules based on reinforcers and attention. Trends Cogn Sci 14:64-71. CrossRef Medline

Sawaki R, Luck SJ, Raymond JE (2015) How attention changes in response to incentives. J Cogn Neurosci 27:2229-2239. CrossRef Medline 
Schacht A, Adler N, Chen P, Guo T, Sommer W (2012) Association with positive outcome induces early effects in event-related brain potentials. Biol Psychol 89:130-136. CrossRef Medline

Seidl KN, Peelen MV, Kastner S (2012) Neural evidence for distracter suppression during visual search in real-world scenes. J Neurosci 32:1181211819. CrossRef Medline

Tamietto M, de Gelder B (2010) Neural bases of the non-conscious perception of emotional signals. Nat Rev Neurosci 11:697-709. CrossRef Medline

Tversky A, Kahneman D (1989) Rational choice and the framing of decisions. New York: Springer.

Tversky A, Kahneman D (1981) The framing of decisions and the psychology of choice. Science 211:453-458. CrossRef Medline

van Koningsbruggen MG, Ficarella SC, Battelli L, Hickey C (2016) Transcranial random-noise stimulation of visual cortex potentiates value-driven attentional capture. Soc Cogn Affect Neurosci 1:1-8. CrossRef Medline

Wang L, Yu H, Zhou X (2013) Interaction between value and perceptual salience in value-driven attentional capture. J Vis 13:5. CrossRef Medline

Wentura D, Müller P, Rothermund K (2014) Attentional capture by evaluative stimuli: gain- and loss-connoting colors boost the additionalsingleton effect. Psychonom Bull Rev 21:701-707. CrossRef Medline

Williams SM, Goldman-Rakic PS (1993) Characterization of the dopaminergic innervation of the primate frontal cortex using a dopaminespecific antibody. Cereb Cortex 3:199-222. CrossRef Medline

Zimmer U, Keppel MT, Poglitsch C, Ischebeck A (2015) ERP evidence for spatial attention being directed away from disgusting locations. Psychophysiology 52:1317-1327. CrossRef Medline 